\title{
Abundant distinct types of solutions for the nervous biological fractional FitzHugh-Nagumo equation via three different sorts of schemes
}

\author{
Abdel-Haleem Abdel-Aty ${ }^{1,2}$, Mostafa M.A. Khater ${ }^{3,4^{*}}$ (D), Dumitru Baleanu ${ }^{5,6,7}$, E.M. Khalil ${ }^{8}$, \\ Jamel Bouslimi $i^{9,10}$ and M. Omri ${ }^{11}$
}

\section{"Correspondence: \\ mostafa.khater2024@yahoo.com \\ ${ }^{3}$ Department of Mathematics, \\ Faculty of Science, Jiangsu \\ University, Zhenjiang 212013, China \\ ${ }^{4}$ Department of Mathematics, Obour Institutes, Cairo 11828, Egypt \\ Full list of author information is \\ available at the end of the article}

\begin{abstract}
The dynamical attitude of the transmission for the nerve impulses of a nervous system, which is mathematically formulated by the Atangana-Baleanu (AB) time-fractional FitzHugh-Nagumo (FN) equation, is computationally and numerically investigated via two distinct schemes. These schemes are the improved Riccati expansion method and B-spline schemes. Additionally, the stability behavior of the analytical evaluated solutions is illustrated based on the characteristics of the Hamiltonian to explain the applicability of them in the model's applications. Also, the physical and dynamical behaviors of the gained solutions are clarified by sketching them in three different types of plots. The practical side and power of applied methods are shown to explain their ability to use on many other nonlinear evaluation equations.
\end{abstract}

MSC: $35 E 05 ; 35 C 08 ; 35 Q 51 ; 37 L 50$

Keywords: Atangana-Baleanu (AB) fractional operator; FitzHugh-Nagumo (FN) equation; Analytical and numerical solutions; Stability characteristic

\section{Introduction}

Nowadays, the study of bio-mathematical models is considered as an original icon in the investigation of the dynamical and physical behavior of many biological models such as DNA [1], viruses [2, 3], the nerve system, the bacteria cell [4, 5] and their distribution, and the transmission of their impulses, and so on. These models are mathematically formulated depending on laboratory experiments and statistics [6-8]. These bio-models are expressed in nonlinear evaluation equations and system with integer and fractional order. However, studying the fractional bio-models is more important than the models with an integer order because of the nonlocal property that appears only in the fractional models [9-11].

The nervous system is one of these bio-models that are attractive to many researchers; it is a sophisticated collection of neurons and nerves [12-14]. The neuron cells transmit

(c) The Author(s) 2020. This article is licensed under a Creative Commons Attribution 4.0 International License, which permits use, sharing, adaptation, distribution and reproduction in any medium or format, as long as you give appropriate credit to the original author(s) and the source, provide a link to the Creative Commons licence, and indicate if changes were made. The images or other third party material in this article are included in the article's Creative Commons licence, unless indicated otherwise in a credit line to the material. If material is not included in the article's Creative Commons licence and your intended use is not permitted by statutory regulation or exceeds the permitted use, you will need to obtain permission directly from the copyright holder. To view a copy of this licence, visit http://creativecommons.org/licenses/by/4.0/. 
signals between different parts of the body. It mostly looks like an electrical wiring system in the human body. According to the National Institute of Health, this system contains two essential components which are the peripheral nervous system and the central nervous system. The brain, nerves, and spinal cord are primary components of the central nervous system [15].

In contrast, the ganglia (clusters of neurons), the sensory neurons, and nerves are primary components of the peripheral nervous system [16, 17]. These nerve cells contact each other and the central nervous system. Functionally, the nervous system has two main subdivisions: the somatic, or voluntary, component and the autonomic, or involuntary, part. There are two types of movement performed by the living body, namely the voluntary action and the inadvertent movement such as blood pressure, respiratory rate, heartbeat, etc., and all these movements are regulated by the autonomic nervous system, according to Merck Manuals $[18,19]$. The somatic system is full of nerves that connect the spinal cord and muscles with the brain that are considered to be a sensory receptor in the skin [20].

The patients with nerve disorders experience functional difficulties according to the Mayo Clinic, which result in conditions such as [ epilepsy, multiple sclerosis (MS), amyotrophic lateral sclerosis (ALS), Huntington's disease, Alzheimer's disease, stroke, transient ischemic attack (TIA), and sub-arachnoid hemorrhage ] [21]. The mathematical model of the transmission for the nerve impulses of a nervous system is the FN equation [22-24] which looks like another form of the Hodgkin-Huxley model [25]

$$
\left\{\begin{array}{l}
\mathcal{Q}_{i}=\varphi_{i}\left(\mathcal{E}_{m}-\mathcal{E}_{i}\right) \\
\mathcal{Q}=\mathcal{M}_{m} \frac{d \mathcal{E}_{m}}{d t}+\varphi_{\mu}\left(\mathcal{E}_{m}-\mathcal{E}_{\mu}\right)+\varphi_{v_{a}}\left(\mathcal{E}_{m}-\mathcal{E}_{v_{a}}\right)+\varphi_{i}\left(\mathcal{E}_{m}-\mathcal{E}_{v_{i}}\right) \\
\mathcal{Q}_{c}=\mathcal{T}_{m} \frac{d \mathcal{L}_{m}}{d t}
\end{array}\right.
$$

where $\mathcal{E}_{v_{a}}, \mathcal{E}_{m}, \varphi_{E}, \mathcal{T}_{m}, \mathcal{E}_{\mu}, \varphi_{i}, \mathcal{E}_{i}$ respectively describe sodium reversal potentials, ion pumps, leak channels, the lipid bilayer, the potassium, the leak conductance per unit area, and membrane potential.

In this context, we study the $\mathrm{AB}$ time-fractional FN equation [26, 27]

$$
\mathcal{N}_{x x}-\mathcal{N}(1-\mathcal{N})(\rho-\mathcal{N})-\mathcal{D}_{t}^{\alpha} \mathcal{N}=0, \quad 0<\alpha<1
$$

where $\rho$ is an arbitrary constant. Equation (2) takes the Newell-Whitehead $(\mathcal{N W})$ equation's form when $\rho=0$.

Recently, many research papers have investigated the analytical and numerical solutions of the time fractional FN equation [28-37] for discovering novel properties of the transmission for the nerve impulses of a nervous system. These solutions are very useful tools for better understanding of the transmission attitude.

In this research paper, the improved Riccati expansion method is applied to the nervous biological fractional FN equation to investigate the analytical solutions of it. Many novel computational solutions are obtained, then they are used to evaluate the initial and boundary conditions. These conditions are employed to handle the numerical solutions of this biological model to show the accuracy of the obtained analytical solutions by calculating the absolute value of error. The obtained solutions are successfully sketched to show the physical and dynamical behavior of these solutions. Moreover, the stability feature of 
solutions is investigated to demonstrate their applicability in its applications where many analytical and numerical schemes have been derived to construct the exact and numerical schemes of this kind of nonlinear evolutions equations [38-49].

The rest of paper is as follows. Section 2 applies computational and numerical schemes [50-56] to the AB time-fractional FN equation for constructing exact and numerical wave solutions. Section 3 illustrates the stability characteristic of the evaluated computational solutions. Section 4 shows, explains, and discusses the relation between our calculated solutions and previously gained solutions by other schemes. Section 5 gives the conclusion.

\section{Application}

This section employs the improved Riccati expansion method and B-spline schemes to find the analytical and numerical solutions. Using the following $\mathrm{AB}$ wave transformation $\mathcal{N}=\mathcal{N}(x, t)=\mathcal{Q}(\mathbb{k}), \mathbb{k}=\frac{(1-\vartheta)\left(\omega t^{-m \vartheta}\right)}{B(\vartheta) \sum_{m=0}^{\infty}\left(-\frac{\vartheta}{1-\vartheta}\right)^{m} \Gamma(1-m \vartheta)}+k x$, where $\lambda, k[56-58]$ are arbitrary constants, yields

$$
k^{2} \mathcal{Q}^{\prime \prime}-\mathcal{Q}(1-\mathcal{Q})(\rho-\mathcal{Q})-\omega \mathcal{Q}^{\prime}=0
$$

Employing the homogeneous balance principles for Eq. (3) yields $\mathcal{Q}^{\prime \prime}, \mathcal{Q}^{3} \Rightarrow n+2=3 n \Rightarrow$ $n=1$.

\subsection{Analytical explicit wave solution}

The general solutions of Eq. (3) based on the improved Riccati expansion method are given by $[57,58]$

$$
\mathcal{Q}(\mathbb{k})=\sum_{i=1}^{n} a_{i} \Lambda(\mathbb{k})^{i}+a_{0}=a_{1} \Lambda(\mathbb{k})+a_{0},
$$

where $a_{i},(i=0,1)$ are arbitrary constants to be determined later. Also, $\Lambda(\mathbb{k})$ satisfies the following ODE:

$$
\Lambda^{\prime}(\mathbb{k})=\delta \Lambda(\mathbb{k})+\sigma \Lambda(\mathbb{k})^{2}+\varrho,
$$

where $\sigma, \varrho, \delta$ are arbitrary constants. Substituting Eq. (4) into Eq. (3), gathering all coefficients with the same power of $\Lambda(\mathbb{k})^{i}(i=-3,-2,-1,0,1,2,3)$, and equating them to zero lead to a system of algebraic equations. Solving this system to get the above-mentioned parameters yields:

\section{Family I:}

$$
\left[a_{0}=\frac{1}{2}(\sqrt{2} \delta k+1), a_{1}=\sqrt{2} k \sigma, \omega=\frac{1}{2}(2 \sqrt{2} k \rho-\sqrt{2} k), \varrho=\frac{2 \delta^{2} k^{2}-1}{8 k^{2} \sigma} .\right]
$$

Consequently, the computational solutions of the $\mathrm{AB}$ time-fractional FN equation are given by the following: 
For $\left[\delta^{2}-4 \sigma \varrho>0 \& \delta \sigma \neq 0\right]$,

$$
\begin{aligned}
\mathcal{N}_{1}(x, t)= & \frac{1}{2}-\frac{k \sqrt{\delta^{2}-4 \sigma \varrho}}{\sqrt{2}} \\
& \times \tanh \left(\frac{1}{2} \sqrt{\delta^{2}-4 \sigma \varrho}\left(k x-\frac{k(2 \rho-1)(\vartheta-1) t^{-m \vartheta}}{\sqrt{2} B(\vartheta) \sum_{m=0}^{\infty}\left(-\frac{\vartheta}{1-\vartheta}\right)^{m} \Gamma(1-m \vartheta)}\right)\right) \\
\mathcal{N}_{2}(x, t)= & \frac{1}{2}-\frac{k \sqrt{\delta^{2}-4 \sigma \varrho}}{\sqrt{2}} \\
& \times \operatorname{coth}\left(\frac{1}{2} \sqrt{\delta^{2}-4 \sigma \varrho}\left(k x-\frac{k(2 \rho-1)(\vartheta-1) t^{-m \vartheta}}{\sqrt{2} B(\vartheta) \sum_{m=0}^{\infty}\left(-\frac{\vartheta}{1-\vartheta}\right)^{m} \Gamma(1-m \vartheta)}\right)\right)
\end{aligned}
$$

For $\left[\delta^{2}-4 \sigma \varrho<0 \& \delta \sigma \neq 0\right]$,

$$
\begin{aligned}
\mathcal{N}_{3}(x, t)= & k \sqrt{2 \sigma \varrho-\frac{\delta^{2}}{2}} \\
& \times \tan \left(\frac{1}{2} \sqrt{4 \sigma \varrho-\delta^{2}}\left(k x-\frac{k(2 \rho-1)(\vartheta-1) t^{-m \vartheta}}{\sqrt{2} B(\vartheta) \sum_{m=0}^{\infty}\left(-\frac{\vartheta}{1-\vartheta}\right)^{m} \Gamma(1-m \vartheta)}\right)\right)+\frac{1}{2}, \\
\mathcal{N}_{4}(x, t)= & \frac{1}{2}-k \sqrt{2 \sigma \varrho-\frac{\delta^{2}}{2}} \\
& \times \cot \left(\frac{1}{2} \sqrt{4 \sigma \varrho-\delta^{2}}\left(k x-\frac{k(2 \rho-1)(\vartheta-1) t^{-m \vartheta}}{\sqrt{2} B(\vartheta) \sum_{m=0}^{\infty}\left(-\frac{\vartheta}{1-\vartheta}\right)^{m} \Gamma(1-m \vartheta)}\right)\right) .
\end{aligned}
$$

For $\left[\delta^{2}-4 \sigma \varrho>0 \& \sigma \varrho \neq 0\right]$,

$$
\begin{aligned}
\mathcal{N}_{5}(x, t) & \\
= & \frac{k}{\sqrt{2}}\left(\delta-\frac{4 \sigma \varrho}{\delta-\sqrt{\delta^{2}-4 \sigma \varrho} \tanh \left(\frac{1}{2} \sqrt{\delta^{2}-4 \sigma \varrho}\left(k x-\frac{k(2 \rho-1)(\vartheta-1) t^{-m \vartheta}}{\sqrt{2} B(\vartheta) \sum_{m=0}^{\infty}\left(-\frac{\vartheta}{1-\vartheta}\right)^{m} \Gamma(1-m \vartheta)}\right)\right)}\right) \\
& +\frac{1}{2}, \\
\mathcal{N}_{6}(x, t) & \frac{k}{\sqrt{2}}\left(\delta-\frac{4 \sigma \varrho}{\delta-\sqrt{\delta^{2}-4 \sigma \varrho} \operatorname{coth}\left(\frac{1}{2} \sqrt{\delta^{2}-4 \sigma \varrho}\left(k x-\frac{k(2 \rho-1)(\vartheta-1) t^{-m \vartheta}}{\sqrt{2} B(\vartheta) \sum_{m=0}^{\infty}\left(-\frac{\vartheta}{1-\vartheta}\right)^{m} \Gamma(1-m \vartheta)}\right)\right)}\right) \\
& +\frac{1}{2} .
\end{aligned}
$$

For $\left[\delta^{2}-4 \sigma \varrho<0 \& \sigma \varrho \neq 0\right]$,

$$
\begin{aligned}
\mathcal{N}_{7}(x, t) & \\
= & \frac{\delta k}{\sqrt{2}}+\frac{1}{2} \\
& -\left[\left(2 \sqrt{2} k \sigma \varrho \cos \left(\frac{1}{2} \sqrt{4 \sigma \varrho-\delta^{2}}\left(k x-\frac{k(2 \rho-1)(\vartheta-1) t^{-m \vartheta}}{\sqrt{2} B(\vartheta) \sum_{m=0}^{\infty}\left(-\frac{\vartheta}{1-\vartheta}\right)^{m} \Gamma(1-m \vartheta)}\right)\right)\right)\right.
\end{aligned}
$$




$$
\begin{aligned}
& /\left(\sqrt{4 \sigma \varrho-\delta^{2}} \sin \left(\frac{1}{2} \sqrt{4 \sigma \varrho-\delta^{2}}\left(k x-\frac{k(2 \rho-1)(\vartheta-1) t^{-m \vartheta}}{\sqrt{2} B(\vartheta) \sum_{m=0}^{\infty}\left(-\frac{\vartheta}{1-\vartheta}\right)^{m} \Gamma(1-m \vartheta)}\right)\right)\right. \\
& \left.\left.+\delta \cosh \left(\frac{1}{2} \sqrt{4 \sigma \varrho-\delta^{2}}\left(k x-\frac{k(2 \rho-1)(\vartheta-1) t^{-m \vartheta}}{\sqrt{2} B(\vartheta) \sum_{m=0}^{\infty}\left(-\frac{\vartheta}{1-\vartheta}\right)^{m} \Gamma(1-m \vartheta)}\right)\right)\right)\right]
\end{aligned}
$$

$\mathcal{N}_{8}(x, t)$

$$
\begin{aligned}
= & \frac{\delta k}{\sqrt{2}}+\frac{1}{2} \\
& +\left[\left(2 \sqrt{2} k \sigma \varrho \sin \left(\frac{1}{2} \sqrt{4 \sigma \varrho-\delta^{2}}\left(k x-\frac{k(2 \rho-1)(\vartheta-1) t^{-m \vartheta}}{\sqrt{2} B(\vartheta) \sum_{m=0}^{\infty}\left(-\frac{\vartheta}{1-\vartheta}\right)^{m} \Gamma(1-m \vartheta)}\right)\right)\right)\right. \\
& /\left(\sqrt{4 \sigma \varrho-\delta^{2}} \cos \left(\frac{1}{2} \sqrt{4 \sigma \varrho-\delta^{2}}\left(k x-\frac{k(2 \rho-1)(\vartheta-1) t^{-m \vartheta}}{\sqrt{2} B(\vartheta) \sum_{m=0}^{\infty}\left(-\frac{\vartheta}{1-\vartheta}\right)^{m} \Gamma(1-m \vartheta)}\right)\right)\right. \\
& \left.\left.-\delta \sinh \left(\frac{1}{2} \sqrt{4 \sigma \varrho-\delta^{2}}\left(k x-\frac{k(2 \rho-1)(\vartheta-1) t^{-m \vartheta}}{\sqrt{2} B(\vartheta) \sum_{m=0}^{\infty}\left(-\frac{\vartheta}{1-\vartheta}\right)^{m} \Gamma(1-m \vartheta)}\right)\right)\right)\right]
\end{aligned}
$$

For $\left[\delta^{2}-4 \sigma \varrho=0 \& \delta \sigma \neq 0\right]$,

$$
\begin{aligned}
& \mathcal{N}_{9}(x, t)=\frac{\delta k\left(\exp \left(\frac{\delta k(2 \rho-1)(\vartheta-1) t^{-m \vartheta}}{\sqrt{2} B(\vartheta) \sum_{m=0}^{\infty}\left(-\frac{\vartheta}{1-\vartheta}\right)^{m} \Gamma(1-m \vartheta)}\right)-\Omega e^{\delta k x}\right)}{\sqrt{2}\left(\exp \left(\frac{\delta k(2 \rho-1)(\vartheta-1) t^{-m \vartheta}}{\sqrt{2} B(\vartheta) \sum_{m=0}^{\infty}\left(-\frac{\vartheta}{1-\vartheta}\right)^{m} \Gamma(1-m \vartheta)}\right)+\Omega e^{\delta k x}\right)}+\frac{1}{2} \\
& \mathcal{N}_{10}(x, t)=\frac{\delta k}{\sqrt{2}}\left(\frac{2 \Omega}{\exp \left(\delta\left(k x-\frac{k(2 \rho-1)(\vartheta-1) t^{-m \vartheta}}{\sqrt{2} B(\vartheta) \sum_{m=0}^{\infty}\left(-\frac{\vartheta}{1-\vartheta}\right)^{m} \Gamma(1-m \vartheta)}\right)\right)+\Omega}-1\right)+\frac{1}{2} .
\end{aligned}
$$

Family II:

$$
\left[a_{1}=-\sqrt{2} k \sigma, \omega=k\left(\sqrt{2}\left(a_{0}-1\right)+\delta k\right), \rho=2 a_{0}+\sqrt{2} \delta k, \varrho=\frac{(-\sqrt{2}) a_{0} \delta k-a_{0}^{2}}{2 k^{2} \sigma} .\right]
$$

Consequently, the computational solutions of the $\mathrm{AB}$ time-fractional FN equation are given by the following:

For $\left[\delta^{2}-4 \sigma \varrho>0 \& \delta \sigma \neq 0\right]$,

$$
\begin{aligned}
& \mathcal{N}_{11}(x, t) \\
& =\frac{k}{\sqrt{2}}\left(\sqrt{\delta^{2}-4 \sigma \varrho}\right. \\
& \left.\quad \times \tanh \left(\frac{1}{2} k \sqrt{\delta^{2}-4 \sigma \varrho}\left(x-\frac{(\vartheta-1)\left(\sqrt{2}\left(a_{0}-1\right)+\delta k\right) t^{-m \vartheta}}{B(\vartheta) \sum_{m=0}^{\infty}\left(-\frac{\vartheta}{1-\vartheta}\right)^{m} \Gamma(1-m \vartheta)}\right)\right)+\delta\right)+a_{0}, \\
& \mathcal{N}_{12}(x, t) \\
& =\frac{k}{\sqrt{2}}\left(\sqrt{\delta^{2}-4 \sigma \varrho}\right. \\
& \left.\quad \times \operatorname{coth}\left(\frac{1}{2} k \sqrt{\delta^{2}-4 \sigma \varrho}\left(x-\frac{(\vartheta-1)\left(\sqrt{2}\left(a_{0}-1\right)+\delta k\right) t^{-m \vartheta}}{B(\vartheta) \sum_{m=0}^{\infty}\left(-\frac{\vartheta}{1-\vartheta}\right)^{m} \Gamma(1-m \vartheta)}\right)\right)+\delta\right)+a_{0} .
\end{aligned}
$$


For $\left[\delta^{2}-4 \sigma \varrho<0 \& \delta \sigma \neq 0\right]$,

$$
\begin{aligned}
& \mathcal{N}_{13}(x, t) \\
& =\frac{k}{\sqrt{2}}\left(\delta-\sqrt{4 \sigma \varrho-\delta^{2}}\right. \\
& \left.\quad \times \tan \left(\frac{1}{2} k \sqrt{4 \sigma \varrho-\delta^{2}}\left(x-\frac{(\vartheta-1)\left(\sqrt{2}\left(a_{0}-1\right)+\delta k\right) t^{-m \vartheta}}{B(\vartheta) \sum_{m=0}^{\infty}\left(-\frac{\vartheta}{1-\vartheta}\right)^{m} \Gamma(1-m \vartheta)}\right)\right)\right)+a_{0}, \\
& \mathcal{N}_{14}(x, t) \\
& =\frac{k}{\sqrt{2}}\left(\sqrt{4 \sigma \varrho-\delta^{2}}\right. \\
& \left.\quad \times \cot \left(\frac{1}{2} k \sqrt{4 \sigma \varrho-\delta^{2}}\left(x-\frac{(\vartheta-1)\left(\sqrt{2}\left(a_{0}-1\right)+\delta k\right) t^{-m \vartheta}}{B(\vartheta) \sum_{m=0}^{\infty}\left(-\frac{\vartheta}{1-\vartheta}\right)^{m} \Gamma(1-m \vartheta)}\right)\right)+\delta\right)+a_{0} .
\end{aligned}
$$

For $\left[\delta^{2}-4 \sigma \varrho>0 \& \sigma \varrho \neq 0\right]$,

$$
\begin{aligned}
& \mathcal{N}_{15}(x, t)=\frac{2 \sqrt{2} k \sigma \varrho}{\delta-\sqrt{\delta^{2}-4 \sigma \varrho} \tanh \left(\frac{1}{2} k \sqrt{\delta^{2}-4 \sigma \varrho}\left(x-\frac{(\vartheta-1)\left(\sqrt{2}\left(a_{0}-1\right)+\delta k\right) t^{-m \vartheta}}{B(\vartheta) \sum_{m=0}^{\infty}\left(-\frac{\vartheta}{1-\vartheta}\right)^{m} \Gamma(1-m \vartheta)}\right)\right)}+a_{0}, \\
& \mathcal{N}_{16}(x, t)=\frac{2 \sqrt{2} k \sigma \varrho}{\delta-\sqrt{\delta^{2}-4 \sigma \varrho} \operatorname{coth}\left(\frac{1}{2} k \sqrt{\delta^{2}-4 \sigma \varrho}\left(x-\frac{(\vartheta-1)\left(\sqrt{2}\left(a_{0}-1\right)+\delta k\right) t^{-m \vartheta}}{B(\vartheta) \sum_{m=0}^{\infty}\left(-\frac{\vartheta}{1-\vartheta}\right)^{m} \Gamma(1-m \vartheta)}\right)\right)}+a_{0} .
\end{aligned}
$$

For $\left[\delta^{2}-4 \sigma \varrho<0 \& \sigma \varrho \neq 0\right]$,

$$
\begin{aligned}
\mathcal{N}_{17}(x, t) & \\
= & a_{0}+\left[\left(2 \sqrt{2} k \sigma \varrho \cos \left(\frac{1}{2} k \sqrt{4 \sigma \varrho-\delta^{2}}\left(x-\frac{(\vartheta-1)\left(\sqrt{2}\left(a_{0}-1\right)+\delta k\right) t^{-m \vartheta}}{B(\vartheta) \sum_{m=0}^{\infty}\left(-\frac{\vartheta}{1-\vartheta}\right)^{m} \Gamma(1-m \vartheta)}\right)\right)\right)\right. \\
& /\left(\sqrt{4 \sigma \varrho-\delta^{2}} \sin \left(\frac{1}{2} k \sqrt{4 \sigma \varrho-\delta^{2}}\left(x-\frac{(\vartheta-1)\left(\sqrt{2}\left(a_{0}-1\right)+\delta k\right) t^{-m \vartheta}}{B(\vartheta) \sum_{m=0}^{\infty}\left(-\frac{\vartheta}{1-\vartheta}\right)^{m} \Gamma(1-m \vartheta)}\right)\right)\right. \\
+ & \left.\left.\delta \cosh \left(\frac{1}{2} k \sqrt{4 \sigma \varrho-\delta^{2}}\left(x-\frac{(\vartheta-1)\left(\sqrt{2}\left(a_{0}-1\right)+\delta k\right) t^{-m \vartheta}}{B(\vartheta) \sum_{m=0}^{\infty}\left(-\frac{\vartheta}{1-\vartheta}\right)^{m} \Gamma(1-m \vartheta)}\right)\right)\right)\right],
\end{aligned}
$$

$\mathcal{N}_{18}(x, t)$

$$
\begin{aligned}
= & a_{0}+\left[\left(2 \sqrt{2} k \sigma \varrho \sin \left(\frac{1}{2} k \sqrt{4 \sigma \varrho-\delta^{2}}\left(x-\frac{(\vartheta-1)\left(\sqrt{2}\left(a_{0}-1\right)+\delta k\right) t^{-m \vartheta}}{B(\vartheta) \sum_{m=0}^{\infty}\left(-\frac{\vartheta}{1-\vartheta}\right)^{m} \Gamma(1-m \vartheta)}\right)\right)\right)\right. \\
& /\left(\delta \sinh \left(\frac{1}{2} k \sqrt{4 \sigma \varrho-\delta^{2}}\left(x-\frac{(\vartheta-1)\left(\sqrt{2}\left(a_{0}-1\right)+\delta k\right) t^{-m \vartheta}}{B(\vartheta) \sum_{m=0}^{\infty}\left(-\frac{\vartheta}{1-\vartheta}\right)^{m} \Gamma(1-m \vartheta)}\right)\right)\right. \\
& \left.\left.-\sqrt{4 \sigma \varrho-\delta^{2}} \cos \left(\frac{1}{2} k \sqrt{4 \sigma \varrho-\delta^{2}}\left(x-\frac{(\vartheta-1)\left(\sqrt{2}\left(a_{0}-1\right)+\delta k\right) t^{-m \vartheta}}{B(\vartheta) \sum_{m=0}^{\infty}\left(-\frac{\vartheta}{1-\vartheta}\right)^{m} \Gamma(1-m \vartheta)}\right)\right)\right)\right] .
\end{aligned}
$$


Abdel-Aty et al. Advances in Difference Equations

(2020) $2020: 476$

Page 7 of 17

For $\left[\delta^{2}-4 \sigma \varrho=0 \& \delta \sigma \neq 0\right]$,

$$
\begin{aligned}
& \mathcal{N}_{19}(x, t)=\frac{\sqrt{2} \delta k \Omega}{\exp \left(\delta(-k)\left(x-\frac{(\vartheta-1)\left(\sqrt{2}\left(a_{0}-1\right)+\delta k\right) t^{-m \vartheta}}{B(\vartheta) \sum_{m=0}^{\infty}\left(-\frac{\vartheta}{1-\vartheta}\right)^{m} \Gamma(1-m \vartheta)}\right)\right)+\Omega}+a_{0}, \\
& \mathcal{N}_{20}(x, t)=\sqrt{2} \delta k\left(1-\frac{\Omega}{\exp \left(\delta k\left(x-\frac{(\vartheta-1)\left(\sqrt{2}\left(a_{0}-1\right)+\delta k\right) t^{-m \vartheta}}{B(\vartheta) \sum_{m=0}^{\infty}\left(-\frac{\vartheta}{1-\vartheta}\right)^{m} \Gamma(1-m \vartheta)}\right)\right)+\Omega}\right)+a_{0} .
\end{aligned}
$$

Family III:

$$
\left[a_{1}=-\sqrt{2} k \sigma, \omega=k\left(\sqrt{2} a_{0}+\delta k\right), \rho=2 a_{0}+\sqrt{2} \delta k-1, \varrho=-\frac{\left(a_{0}-1\right)\left(a_{0}+\sqrt{2} \delta k-1\right)}{2 k^{2} \sigma} .\right]
$$

Consequently, the computational solutions of the $\mathrm{AB}$ time-fractional FN equation are given by the following:

For $\left[\delta^{2}-4 \sigma \varrho>0 \& \delta \sigma \neq 0\right]$,

$$
\begin{aligned}
\mathcal{N}_{21}(x, t) & \\
= & \frac{k}{\sqrt{2}}\left(\sqrt{\delta^{2}-4 \sigma \varrho}\right. \\
& \left.\quad \times \tanh \left(\frac{1}{2} k \sqrt{\delta^{2}-4 \sigma \varrho}\left(x-\frac{(\vartheta-1)\left(\sqrt{2} a_{0}+\delta k\right) t^{-m \vartheta}}{B(\vartheta) \sum_{m=0}^{\infty}\left(-\frac{\vartheta}{1-\vartheta}\right)^{m} \Gamma(1-m \vartheta)}\right)\right)+\delta\right)+a_{0}, \\
\mathcal{N}_{22}(x, t) & \frac{k}{\sqrt{2}}\left(\sqrt{\delta^{2}-4 \sigma \varrho}\right. \\
& \left.\quad \times \operatorname{coth}\left(\frac{1}{2} k \sqrt{\delta^{2}-4 \sigma \varrho}\left(x-\frac{(\vartheta-1)\left(\sqrt{2} a_{0}+\delta k\right) t^{-m \vartheta}}{B(\vartheta) \sum_{m=0}^{\infty}\left(-\frac{\vartheta}{1-\vartheta}\right)^{m} \Gamma(1-m \vartheta)}\right)\right)+\delta\right)+a_{0} .
\end{aligned}
$$

For $\left[\delta^{2}-4 \sigma \varrho<0 \& \delta \sigma \neq 0\right]$,

$$
\begin{aligned}
& \mathcal{N}_{23}(x, t) \\
& =\frac{k}{\sqrt{2}}\left(\delta-\sqrt{4 \sigma \varrho-\delta^{2}}\right. \\
& \left.\quad \times \tan \left(\frac{1}{2} k \sqrt{4 \sigma \varrho-\delta^{2}}\left(x-\frac{(\vartheta-1)\left(\sqrt{2} a_{0}+\delta k\right) t^{-m \vartheta}}{B(\vartheta) \sum_{m=0}^{\infty}\left(-\frac{\vartheta}{1-\vartheta}\right)^{m} \Gamma(1-m \vartheta)}\right)\right)\right)+a_{0}, \\
& \mathcal{N}_{24}(x, t) \\
& =\frac{k}{\sqrt{2}}\left(\sqrt{4 \sigma \varrho-\delta^{2}}\right. \\
& \left.\quad \times \cot \left(\frac{1}{2} k \sqrt{4 \sigma \varrho-\delta^{2}}\left(x-\frac{(\vartheta-1)\left(\sqrt{2} a_{0}+\delta k\right) t^{-m \vartheta}}{B(\vartheta) \sum_{m=0}^{\infty}\left(-\frac{\vartheta}{1-\vartheta}\right)^{m} \Gamma(1-m \vartheta)}\right)\right)+\delta\right)+a_{0} .
\end{aligned}
$$


For $\left[\delta^{2}-4 \sigma \varrho>0 \& \sigma \varrho \neq 0\right]$,

$$
\begin{aligned}
& \mathcal{N}_{25}(x, t)=\frac{2 \sqrt{2} k \sigma \varrho}{\delta-\sqrt{\delta^{2}-4 \sigma \varrho} \tanh \left(\frac{1}{2} k \sqrt{\delta^{2}-4 \sigma \varrho}\left(x-\frac{(\vartheta-1)\left(\sqrt{2} a_{0}+\delta k\right) t^{-m \vartheta}}{B(\vartheta) \sum_{m=0}^{\infty}\left(-\frac{\vartheta}{1-\vartheta}\right)^{m} \Gamma(1-m \vartheta)}\right)\right)}+a_{0}, \\
& \mathcal{N}_{26}(x, t)=\frac{2 \sqrt{2} k \sigma \varrho}{\delta-\sqrt{\delta^{2}-4 \sigma \varrho} \operatorname{coth}\left(\frac{1}{2} k \sqrt{\delta^{2}-4 \sigma \varrho}\left(x-\frac{(\vartheta-1)\left(\sqrt{2} a_{0}+\delta k\right) t^{-m \vartheta}}{B(\vartheta) \sum_{m=0}^{\infty}\left(-\frac{\vartheta}{1-\vartheta}\right)^{m} \Gamma(1-m \vartheta)}\right)\right.}+a_{0} .
\end{aligned}
$$

For $\left[\delta^{2}-4 \sigma \varrho<0 \& \sigma \varrho \neq 0\right]$,

$$
\begin{aligned}
\mathcal{N}_{27}(x, t) & \\
= & a_{0}+\left[\left(2 \sqrt{2} k \sigma \varrho \cos \left(\frac{1}{2} k \sqrt{4 \sigma \varrho-\delta^{2}}\left(x-\frac{(\vartheta-1)\left(\sqrt{2} a_{0}+\delta k\right) t^{-m \vartheta}}{B(\vartheta) \sum_{m=0}^{\infty}\left(-\frac{\vartheta}{1-\vartheta}\right)^{m} \Gamma(1-m \vartheta)}\right)\right)\right)\right. \\
& /\left(\sqrt{4 \sigma \varrho-\delta^{2}} \sin \left(\frac{1}{2} k \sqrt{4 \sigma \varrho-\delta^{2}}\left(x-\frac{(\vartheta-1)\left(\sqrt{2} a_{0}+\delta k\right) t^{-m \vartheta}}{B(\vartheta) \sum_{m=0}^{\infty}\left(-\frac{\vartheta}{1-\vartheta}\right)^{m} \Gamma(1-m \vartheta)}\right)\right)\right. \\
& \left.\left.+\delta \cosh \left(\frac{1}{2} k \sqrt{4 \sigma \varrho-\delta^{2}}\left(x-\frac{(\vartheta-1)\left(\sqrt{2} a_{0}+\delta k\right) t^{-m \vartheta}}{B(\vartheta) \sum_{m=0}^{\infty}\left(-\frac{\vartheta}{1-\vartheta}\right)^{m} \Gamma(1-m \vartheta)}\right)\right)\right)\right] \\
\mathcal{N}_{28}(x, t) & \\
= & a_{0}+\left[\left(2 \sqrt{2} k \sigma \varrho \sin \left(\frac{1}{2} k \sqrt{4 \sigma \varrho-\delta^{2}}\left(x-\frac{(\vartheta-1)\left(\sqrt{2} a_{0}+\delta k\right) t^{-m \vartheta}}{B(\vartheta) \sum_{m=0}^{\infty}\left(-\frac{\vartheta}{1-\vartheta}\right)^{m} \Gamma(1-m \vartheta)}\right)\right)\right)\right. \\
& /\left(\delta \sinh \left(\frac{1}{2} k \sqrt{4 \sigma \varrho-\delta^{2}}\left(x-\frac{(\vartheta-1)\left(\sqrt{2} a_{0}+\delta k\right) t^{-m \vartheta}}{B(\vartheta) \sum_{m=0}^{\infty}\left(-\frac{\vartheta}{1-\vartheta}\right)^{m} \Gamma(1-m \vartheta)}\right)\right)\right. \\
& \left.\left.-\sqrt{4 \sigma \varrho-\delta^{2}} \cos \left(\frac{1}{2} k \sqrt{4 \sigma \varrho-\delta^{2}}\left(x-\frac{(\vartheta-1)\left(\sqrt{2} a_{0}+\delta k\right) t^{-m \vartheta}}{B(\vartheta) \sum_{m=0}^{\infty}\left(-\frac{\vartheta}{1-\vartheta}\right)^{m} \Gamma(1-m \vartheta)}\right)\right)\right)\right] .
\end{aligned}
$$

For $\left[\delta^{2}-4 \sigma \varrho=0 \& \delta \sigma \neq 0\right]$,

$$
\begin{aligned}
& \mathcal{N}_{29}(x, t)=\frac{\sqrt{2} \delta k \Omega}{\exp \left(\delta(-k)\left(x-\frac{\vartheta-1)\left(\sqrt{2} a_{0}+\delta k\right)^{-m \vartheta}}{B(\vartheta) \sum_{m=0}^{\infty}\left(-\frac{\vartheta}{1-\vartheta}\right)^{m} \Gamma(1-m \vartheta)}\right)+\Omega\right.}+a_{0}, \\
& \mathcal{N}_{30}(x, t)=\sqrt{2} \delta k\left(1-\frac{\Omega}{\exp \left(\delta k\left(x-\frac{(\vartheta-1)\left(\sqrt{2} a_{0}+\delta k\right) t^{-m \vartheta}}{B(\vartheta) \sum_{m=0}^{\infty}\left(-\frac{\vartheta}{1-\vartheta}\right)^{t} \Gamma(1-m \vartheta)}\right)+\Omega\right.}\right)+a_{0} .
\end{aligned}
$$

\subsection{Numerical simulation}

In this section, the B-spline scheme is applied to the fractional biological FN equation to evaluate the numerical solution of it and also to show the accuracy of the gained analytical solutions that are evaluated in Sect. 2.1 by employing the improved Riccati expansion method under the following conditions on Eq. (5):

$$
\left[\delta=5, k=\frac{1}{\sqrt{2}}, \rho=-1, \sigma=1, \omega=-\frac{3}{2}, \varrho=6 .\right]
$$

These conditions allow applying the B-spline family in the following forms. 
Table 1 Analytical, numerical, and absolute values in different values of $\mathbb{k}$ via cubic B-spline scheme, showing the accuracy of the obtained analytical solution

\begin{tabular}{llll}
\hline Value of $\mathbb{k}$ & Approximate values & Analytical values & Absolute values of error \\
\hline 0 & 0.5 & 0.5 & $5.55112 \times 10^{-17}$ \\
0.001 & 0.499751 & 0.49975 & $8.45294 \times 10^{-7}$ \\
0.002 & 0.499502 & 0.4995 & $1.50299 \times 10^{-6}$ \\
0.003 & 0.499252 & 0.49925 & $1.97301 \times 10^{-6}$ \\
0.004 & 0.499002 & 0.499 & $2.25524 \times 10^{-6}$ \\
0.005 & 0.498752 & 0.49875 & $2.3496 \times 10^{-6}$ \\
0.006 & 0.498502 & 0.4985 & $2.25598 \times 10^{-6}$ \\
0.007 & 0.498252 & 0.49825 & $1.97431 \times 10^{-6}$ \\
0.008 & 0.498002 & 0.498 & $1.50449 \times 10^{-6}$ \\
0.009 & 0.497751 & 0.49775 & $8.46413 \times 10^{-7}$ \\
0.01 & 0.4975 & 0.4975 & $5.55112 \times 10^{-17}$ \\
\hline
\end{tabular}

\subsubsection{Cubic-spline}

This scheme formulates the general solution of Eq. (2) in the form

$$
\mathcal{N}(\mathbb{k})=\sum_{J=-1}^{m+1} \mho_{\jmath} \partial_{\jmath},
$$

where $\mho_{J}, \mho_{J}$ are given in the following mathematical forms, respectively:

$$
\mathcal{L N}(\mathbb{k})=f\left(\mathbb{k}_{\jmath}, \mathcal{V}\left(\mathbb{k}_{J}\right)\right), \quad(J=0,1, \ldots, m)
$$

and

$\partial_{J}(\mathbb{k})$

$$
=\frac{1}{6 \hbar^{3}} \begin{cases}\left(\mathbb{k}-\mathbb{k}_{J-2}\right)^{3}, & \mathbb{k} \in\left[\mathbb{k}_{J-2}, \mathbb{k}_{J-1}\right], \\ -3\left(\mathbb{k}-\mathbb{k}_{J-1}\right)^{3}+3 \hbar\left(\mathbb{k}_{-}-\mathbb{k}_{J-1}\right)^{2}+3 \hbar^{2}\left(\mathbb{k}_{-}-\mathbb{k}_{J-1}\right)+\hbar^{3}, & \mathbb{k} \in\left[\mathbb{k}_{J-1}, \mathbb{k}_{J}\right], \\ -3\left(\mathbb{k}_{J+1}-\mathbb{k}\right)^{3}+3 \hbar\left(\mathbb{k}_{J+1}-\mathbb{k}\right)^{2}+3 \hbar^{2}\left(\mathbb{k}_{i+1}-\mathbb{k}\right)+\hbar^{3}, & \mathbb{k} \in\left[\mathbb{k}_{i}, \mathbb{k}_{i+1}\right], \\ \left(\mathbb{k}_{i+2}-\mathbb{k}\right)^{3}, & \mathbb{k} \in\left[\mathbb{k}_{i+1}, \mathbb{k}_{i+2}\right], \\ 0, & \text { Otherwise, }\end{cases}
$$

where $J \in[-2, m+2]$. Thus, we obtain

$$
\mathcal{N}_{J}(\mathbb{k})=\mho_{\jmath-1}+4 \mho_{\jmath}+\mho_{\jmath+1} .
$$

Substituting Eq. (37) into Eq. (3) yields $(m+3)$ of equations. Using Mathematica 11.3 to solve this system to get the value of $\mho_{J}$ leads to the following analytical, numerical values under the different values of $\mathbb{k}$ in Table 1 .

\subsubsection{Quantic-spline}

This scheme formulates the general solution of Eq. (2) in the form

$$
\mathcal{N}(\mathbb{k})=\sum_{J=-1}^{m+1} \mho_{J} \partial_{J},
$$


Table 2 Analytical, numerical, and absolute values in different values of $\mathbb{k}$ via quantic B-spline scheme, showing the accuracy of the obtained analytical solution

\begin{tabular}{llll}
\hline Value of $\mathbb{k}$ & Approximate values & Analytical values & Absolute values of error \\
\hline 0 & 0.5 & 0.5 & 0 \\
0.001 & 0.499751 & 0.49975 & $5.70289 \times 10^{-7}$ \\
0.002 & 0.499501 & 0.4995 & $1.30164 \times 10^{-6}$ \\
0.003 & 0.499252 & 0.49925 & $1.75184 \times 10^{-6}$ \\
0.004 & 0.499002 & 0.499 & $2.03943 \times 10^{-6}$ \\
0.005 & 0.498752 & 0.49875 & $2.13195 \times 10^{-6}$ \\
0.006 & 0.498502 & 0.4985 & $2.0401 \times 10^{-6}$ \\
0.007 & 0.498252 & 0.49825 & $1.75299 \times 10^{-6}$ \\
0.008 & 0.498001 & 0.498 & $1.30293 \times 10^{-6}$ \\
0.009 & 0.497751 & 0.49775 & $5.71006 \times 10^{-7}$ \\
0.01 & 0.4975 & 0.4975 & $5.55112 \times 10^{-17}$ \\
\hline
\end{tabular}

where $\mho_{J}, \partial_{J}$ are given in the following mathematical forms, respectively:

$$
\mathcal{L N}(\mathbb{k})=f\left(\mathbb{k}_{\jmath}, \mathcal{N}\left(\mathbb{k}_{J}\right)\right), \quad(j=0,1, \ldots, n)
$$

and

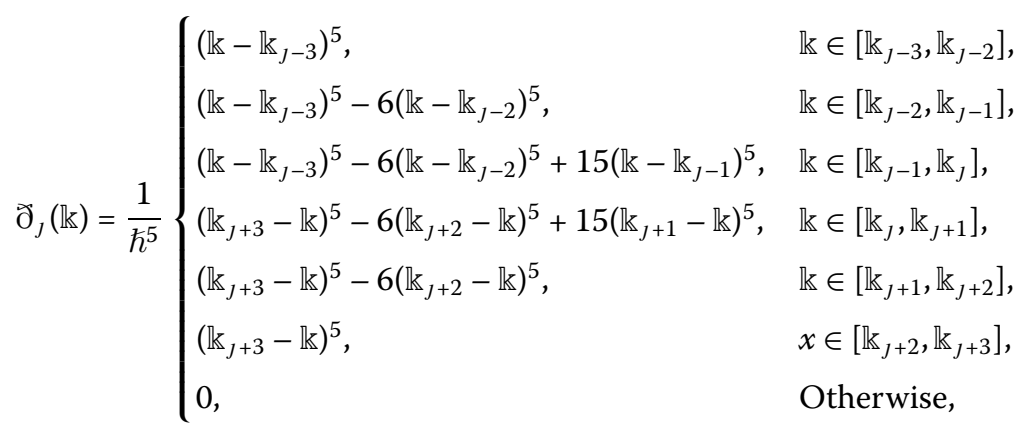

where $\jmath \in[-2, m+2]$. Thus, we obtain

$$
\mathcal{N}_{J}(\mathbb{k})=\mho_{J-2}+26 \mho_{J-1}+66 \mho_{\jmath}+26 \mho_{\jmath+1}+\mho_{\jmath+2}
$$

Substituting Eq. (40) into Eq. (3) yields $(m+5)$ of equations. Using Mathematica 11.3 to solve this system to get the value of $\mho_{J}$ leads to the following analytical, numerical values under the different values of $\mathbb{k}$ in Table 2 .

\subsubsection{Septic-spline}

This scheme formulates the general solution of Eq. (2) in the form

$$
\mathcal{N}(\mathbb{k})=\sum_{J=-1}^{n+1} \mho_{J} \partial_{J},
$$

where $\mho_{J}, \partial_{J}$ are given in the following mathematical forms, respectively:

$$
\mathcal{L N}(\mathbb{k})=\mathcal{F}\left(\mathbb{k}_{J}, \mathcal{N}\left(\mathbb{k}_{J}\right)\right), \quad(J=0,1, \ldots, m)
$$


Table 3 Analytical, numerical, and absolute values in different values of $\mathbb{k}$ via septic B-spline scheme, showing the accuracy of the obtained analytical solution

\begin{tabular}{llll}
\hline Value of $\mathbb{k}$ & Approximate values & Analytical values & Absolute values of error \\
\hline 0 & 0.5 & 0.5 & 0 \\
0.001 & 0.499751 & 0.49975 & $7.50007 \times 10^{-7}$ \\
0.002 & 0.499502 & 0.4995 & $1.59591 \times 10^{-6}$ \\
0.003 & 0.499252 & 0.49925 & $1.97751 \times 10^{-6}$ \\
0.004 & 0.499002 & 0.499 & $2.30056 \times 10^{-6}$ \\
0.005 & 0.498752 & 0.49875 & $2.37162 \times 10^{-6}$ \\
0.006 & 0.498502 & 0.4985 & $2.30133 \times 10^{-6}$ \\
0.007 & 0.498252 & 0.49825 & $1.9788 \times 10^{-6}$ \\
0.008 & 0.498002 & 0.498 & $1.59754 \times 10^{-6}$ \\
0.009 & 0.497751 & 0.49775 & $7.50962 \times 10^{-7}$ \\
0.01 & 0.4975 & 0.4975 & $5.55112 \times 10^{-17}$ \\
\hline
\end{tabular}

and

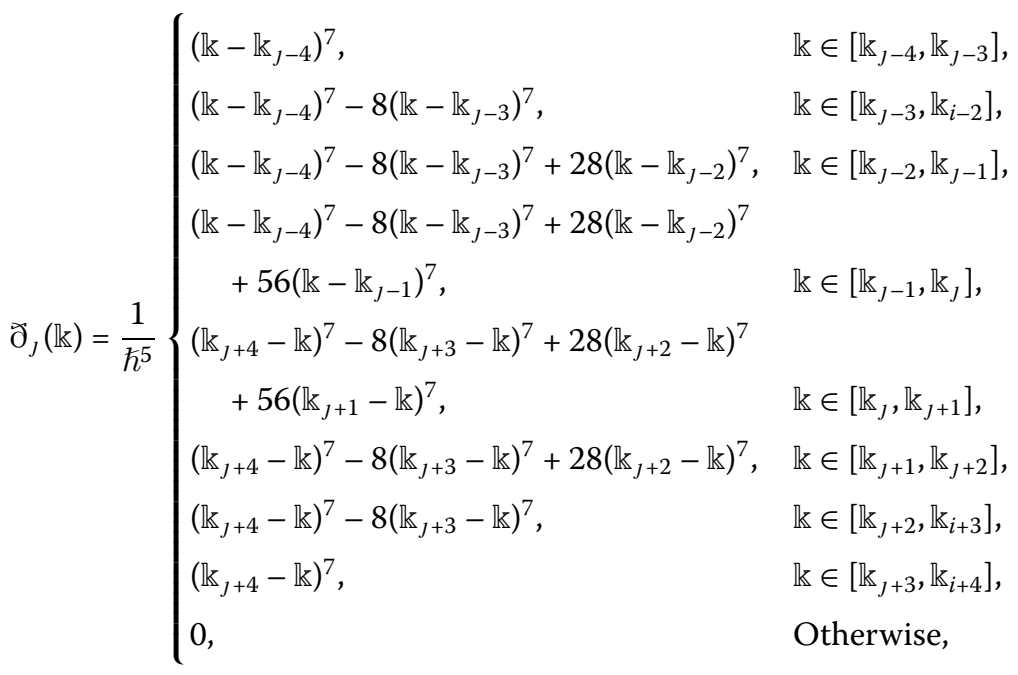

where $j \in[-3, m+3]$. Thus, we obtain

$$
\mathcal{N}_{J}(\mathbb{k})=\mho_{J-3}+120 \mho_{J-2}+1191 \mho_{J-1}+2416 \mho_{J}+1191 \mho_{J+1}+120 \mho_{J+2}+\mho_{J+3}
$$

Substituting Eq. (43) into Eq. (3) yields $(m+7)$ of equations. Using Mathematica 11.3 to solve this system to get the value of $\mho_{J}$ leads to the following analytical, numerical values under the different values of $\mathbb{k}$ in Table 3 .

\section{Stability characteristic}

Investigation of the stability of the obtained analytical solutions by employing the properties of the Hamiltonian system that gives the momentum $\Xi$ in the form

$$
\Xi=\frac{1}{2} \int_{-v}^{v} \mathcal{N}^{2}(\mathbb{k}) d \mathbb{k}
$$

leads to the stable condition of the solution given by

$$
\operatorname{Re}\left(\frac{\partial \Xi}{\partial \omega}\right)>0,
$$


where $\omega$ is the wave velocity. Thus, the investigation of the stability characteristic for Eq. (5) is formulated as follows:

$$
\begin{aligned}
\Xi= & \frac{1}{\omega^{2}}\left[(\sqrt{2}-i \sqrt{10} \omega) \operatorname{Li}_{2}\left(-e^{i \omega \sqrt{5}+\frac{5}{\sqrt{2}}}\right)-\sqrt{2} \operatorname{Li}_{3}\left(-e^{\omega \sqrt{5}+\frac{5}{\sqrt{2}}}\right)-\sqrt{2} \operatorname{Li}_{3}\left(-e^{i \sqrt{5} \omega-\frac{5}{\sqrt{2}}}\right)\right. \\
& +\sqrt{2}(\sqrt{5} \omega-1) \operatorname{Li}_{2}\left(-e^{\omega \sqrt{5}+\frac{5}{\sqrt{2}}}\right)+\sqrt{2}(1-\sqrt{5} \omega) \operatorname{Li}_{2}\left(-e^{\sqrt{5} \omega-\frac{5}{\sqrt{2}}}\right) \\
& +\sqrt{2}(-1+i \sqrt{5} \omega) \operatorname{Li}_{2}\left(-e^{i \sqrt{5} \omega-\frac{5}{\sqrt{2}}}\right)+\sqrt{2} \operatorname{Li}_{3}\left(-e^{\sqrt{5} \omega-\frac{5}{\sqrt{2}}}\right)+\sqrt{2} \operatorname{Li}_{3}\left(-e^{i \omega \sqrt{5}+\frac{5}{\sqrt{2}}}\right) \\
& +50 \omega^{2}+\sqrt{5} \omega\left(-i \sqrt{2} \log \left(e^{\frac{5}{\sqrt{2}}}+e^{i \sqrt{5} \omega}\right)-\sqrt{2} \log \left(e^{\sqrt{5} \omega+\frac{5}{\sqrt{2}}}+1\right)\right. \\
& \left.\left.+\sqrt{2} \log \left(e^{\sqrt{5} \omega}+e^{\frac{5}{\sqrt{2}}}\right)+i \sqrt{2} \log \left(1+e^{\frac{5}{\sqrt{2}}+i \sqrt{5} \omega}\right)+(-5+5 i)\right)\right]
\end{aligned}
$$

and thus

$$
\operatorname{Re}\left(\left.\frac{\partial \Xi}{\partial \omega}\right|_{\omega=-\frac{3}{2}}\right)=70.4226>0
$$

This result shows that the stable property of Eq. (5) is accomplished. Therefore, applying the same steps to the other analytical solution explains the stability characteristic of each of them.

\section{Discussion}

Studying the novelty of our solutions is shown in this section by giving more explanation of them and confirming the comparison between our solutions and those obtained in the previous article. Our investigation has two main steps, which are studying the analytical solutions and then surveying the numerical solutions. This process takes the following steps:

\section{Obtained analytical solutions}

- Using a new fractional operator [ Atangana-Baleanu derivative operator] for the first time to transform the nervous biological fractional FN equation into the ordinary differential equation.

- Applying the improved Riccati expansion method to the obtained ODE leads to many analytical solutions of this model.

- Comparing the obtained solutions with the previous ones in the following steps:

(a) In [26], Dumitru Baleanu et al. used the extended simplest equation method and the sinh-cosh expansion method to find the analytical wave solutions of the FN model with the integer order. Some of their solutions are equal to our obtained solutions such as [26, Eq. (10)] is equal to Eq. (5) when $\left[\vartheta=1, k=\frac{\sqrt{2\left(\delta^{2}-4 \sigma \varrho\right)}}{2}.\right]$

(b) In [27], Abdel-Haleem Abdel-Aty et al. employed the modified Khater $(\mathrm{mK})$ method and B-spline schemes to find the analytical and numerical schemes of the FitzHugh-Nagumo (FN) equation with the integer order. Some of their solutions are equal to our obtained solutions such as Eq. (7, [27]) is equal to Eq. (5) when $\left[\vartheta=1, k=\frac{\sqrt{2\left(\delta^{2}-4 \sigma \varrho\right)}}{2}\right.$.]

2. Obtained numerical solutions 
Applying the B-spline schemes on the fractional biological FN model shows the accuracy of the obtained analytical solutions, but it also explains the superiority of the cubic B-spline scheme over the other two applied methods: the absolute value of error obtained by using it is smaller than the absolute value of error calculated by the other two applied schemes. This accuracy of the cubic-B-spline is shown in Fig. 6.

\section{Conclusion}

This paper has successfully performed the improved Riccati expansion method for constructing the exact traveling wave solutions of the nervous biological fractional FN equation that have been represented in Figs. 1, 2. These solutions have been used to evaluate the initial and boundary conditions that have allowed applying the B-spline collection schemes (cubic, quantic, and septic). Referring to these numerical schemes has shown the absolute value of error between the obtained exact and numerical solutions. These values have explained the accuracy of the obtained solutions as shown in Figs. 3, 4, 5. The stability property of the obtained solutions has been investigated based on the Hamiltonian system's features and their ability to use into the biological model's applications. Three- and two-dimensional and contour plots have been given for the obtained exact and numerical solutions to show the physical and dynamical behavior of these solutions. The comparison between the obtained solutions and the previous solutions was shown to explain the novelty of our research.
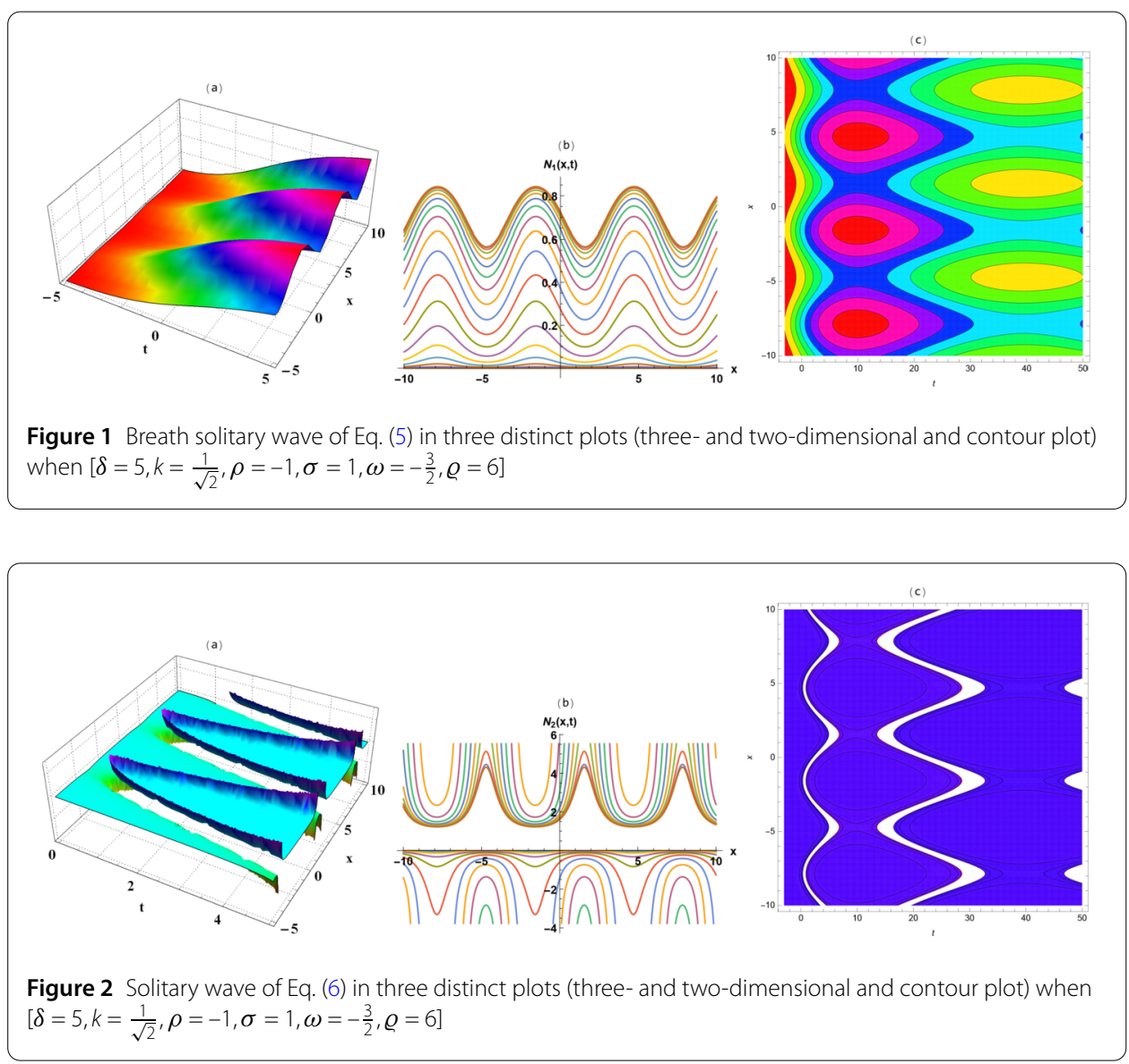

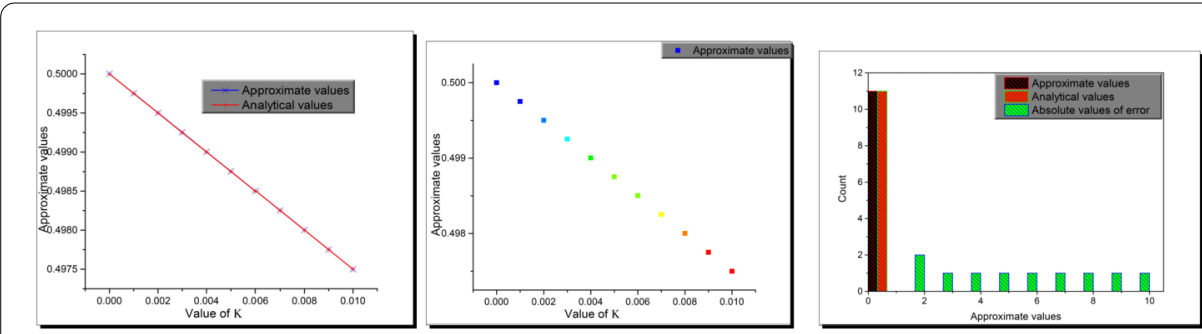

Figure 3 A comparison representation between analytical and numerical solutions of Eq. (2) according to the cubic-B-spline simulation

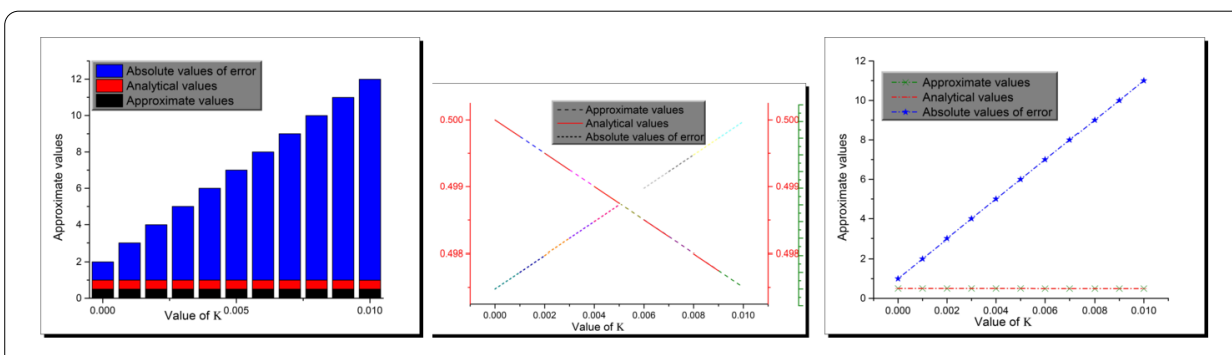

Figure 4 A comparison representation between analytical and numerical solutions of Eq. (1) according to the quantic-B-spline simulation

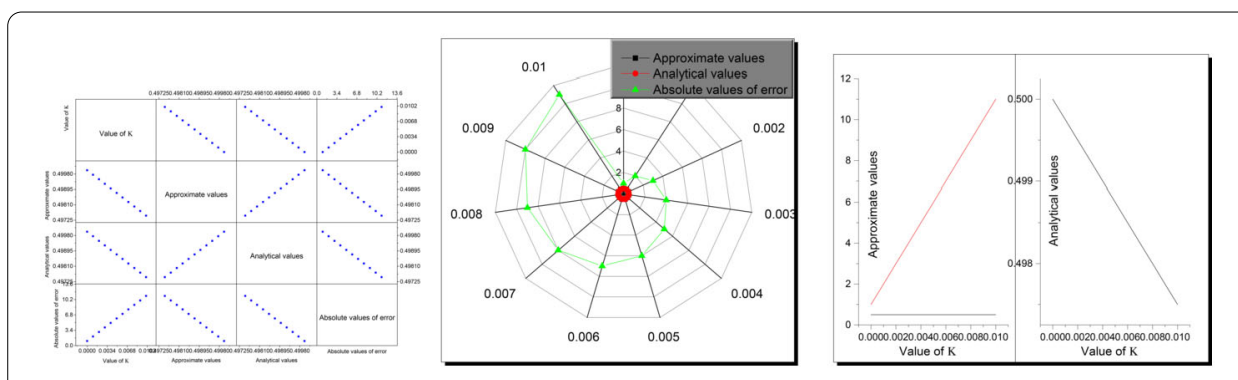

Figure 5 A comparison representation between analytical and numerical solutions of Eq. (1) according to the septic-B-spline simulation

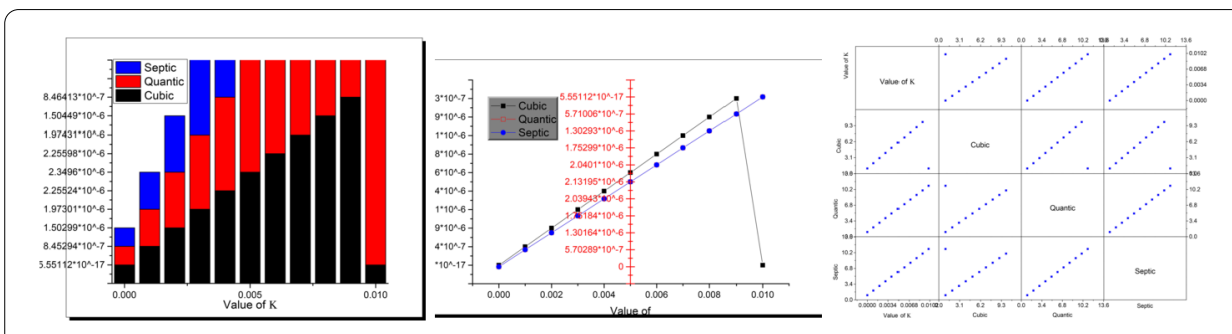

Figure 6 A comparison representation of absolute value of error of Eq. (2) according to the obtained values via cubic, quantic, and septic B-spline schemes

This project was funded by the Deanship of Scientific Research (DSR), King Abdulaziz University, Jeddah, under grant No. (D-672-305-1441). The authors, therefore, gratefully acknowledge DSR technical and financial support. 
Funding

This project was funded by the Deanship of Scientific Research (DSR), King Abdulaziz University, Jeddah, under grant No. (D-672-305-1441). The authors, therefore, gratefully acknowledge DSR technical and financial support.

\section{Availability of data and materials}

Not applicable.

\section{Competing interests}

The authors declare that they have no competing interests.

\section{Authors' contributions}

All authors conceived of the study, participated in its design and coordination, drafted the manuscript, participated in the sequence alignment, and read and approved the final manuscript.

\section{Author details}

${ }^{1}$ Department of Physics, College of Sciences, University of Bisha, P.O. Box 344, Bisha 61922, Saudi Arabia. ${ }^{2}$ Physics Department, Faculty of Science, Al-Azhar University, Assiut 71524, Egypt. ${ }^{3}$ Department of Mathematics, Faculty of Science, Jiangsu University, Zhenjiang 212013, China. ${ }^{4}$ Department of Mathematics, Obour Institutes, Cairo 11828, Egypt. ${ }^{5}$ Department of Mathematics, Cankaya University, Ankara, Turkey. ${ }^{6}$ Institute of Space Sciences, Magurele-Bucharest, Romania. ${ }^{7}$ Department of Medical Research, China Medical University Hospital, China Medical University, Taichung, Taiwan. ${ }^{8}$ Department of Mathematics, Faculty of Science, Taif University, Taif 888, Saudi Arabia. ${ }^{9}$ Department of Engineering Physics and Instrumentation, National Institute of Applied Sciences and Technology, Carthage University, Tunis, Tunisia. ${ }^{10}$ Department of Physics, Faculty of Science, Taif University, Taif 888, Saudi Arabia. ${ }^{11}$ Deanship of Scientific Research, King Abdulaziz University, Jeddah, Saudi Arabia.

\section{Publisher's Note}

Springer Nature remains neutral with regard to jurisdictional claims in published maps and institutional affiliations.

Received: 1 May 2020 Accepted: 23 July 2020 Published online: 09 September 2020

\section{References}

1. Sepehri, A.: A mathematical model for DNA. Int. J. Geom. Methods Mod. Phys. 14(11), 1750152 (2017)

2. Kalemera, M., Mincheva, D., Grove, J., Illingworth, C.J.: Building a mechanistic mathematical model of hepatitis $C$ virus entry. PLoS Comput. Biol. 15(3), e1006905 (2019)

3. Agusto, F.B., Bewick, S., Fagan, W.: Mathematical model of Zika virus with vertical transmission. Infect. Dis. Model. 2(2), 244-267 (2017)

4. Prindle, A., Liu, J., Asally, M., Garcia-Ojalvo, J., Suel, G.: A novel bacterial cell to cell communication mechanism Biophys. J. 114(3), 335a (2018)

5. Bai, H., Cochet, N., Pauss, A., Lamy, E.: Bacteria cell properties and grain size impact on bacteria transport and deposition in porous media. Colloids Surf. B, Biointerfaces 139, 148-155 (2016)

6. Dawson, D., Darwent, D., Roach, G.D.: How should a bio-mathematical model be used within a fatigue risk management system to determine whether or not a working time arrangement is safe? Accid. Anal. Prev. 99, 469-473 (2017)

7. Geng, C., Paganetti, H., Grassberger, C.: Prediction of treatment response for combined chemo-and radiation therapy for non-small cell lung cancer patients using a bio-mathematical model. Sci. Rep. 7(1), 1-12 (2017)

8. Dawson, D., Darwent, D., Roach, G.D.: How should a bio-mathematical model be used within a fatigue risk management system to determine whether or not a working time arrangement is safe. Accid. Anal. Prev. 99, 469-473 (2017)

9. Moaddy, K., Freihat, A., Al-Smadi, M., Abuteen, E., Hashim, I.: Numerical investigation for handling fractional-order Rabinovich-Fabrikant model using the multistep approach. Soft Comput. 22(3), 773-782 (2018)

10. Farman, M., Usman, M., Ahmad, A., Ahmad, M.: Mathematical analysis of fractional order co-infection TB and HIV model. Int. J. Anal. Appl. 18(1), 16-32 (2019)

11. Tajadodi, H.: Numerical solutions of mathematical model on fractional Lotka-Volterra equations. In: 1st Annual National Conference on Biomathematics, p. 120 (2019)

12. Geronikolou, S., Chrousos, G., Albanopoulos, K., Cokkinos, D., Kanaka-Gantenbein, C.: Autonomic nervous system-inflammation link: a new independent mechanism for homeostasis. In: 57th Annual ESPE, vol. 89. European Society for Paediatric Endocrinology, Athens (2018)

13. Zeisel, A., Hochgerner, H., Lönnerberg, P., Johnsson, A., Memic, F., Van Der Zwan, J., Häring, M., Braun, E., Borm, L.E., La Manno, G., et al.: Molecular architecture of the mouse nervous system. Cell 174(4), 999-1014 (2018)

14. Louis, D.N., Perry, A., Reifenberger, G., Von Deimling, A., Figarella-Branger, D., Cavenee, W.K., Ohgaki, H., Wiestler, O.D., Kleihues, P., Ellison, D.W.: The 2016 World Health Organization classification of tumors of the central nervous system: a summary. Acta Neuropathol. 131(6), 803-820 (2016)

15. Anderson, M.A., Burda, J.E., Ren, Y., Ao, Y., O'Shea, T.M., Kawaguchi, R., Coppola, G., Khakh, B.S., Deming, T.J., Sofroniew, M.V.: Astrocyte scar formation aids central nervous system axon regeneration. Nature 532(7598), 195 (2016)

16. Allen, E., Coote, J.H., Grubb, B.D., Batten, T.F., Pauza, D.H., Ng, G.A., Brack, K.E.: Electrophysiological effects of nicotinic and electrical stimulation of intrinsic cardiac ganglia in the absence of extrinsic autonomic nerves in the rabbit heart. Heart Rhythm 15(11), 1698-1707 (2018)

17. Matchen, T., Moehlis, J.: Real-time stabilization of neurons into clusters. In: 2017 American Control Conference (ACC), pp. 2805-2810. IEEE, Seattle (2017)

18. Bullers, K.: Merck manuals. J. Med. Libr. Assoc. 104(4), 369 (2016) 
19. Zhou, P., Grady, S.C., Chen, G.: How the built environment affects change in older people's physical activity: a mixed-methods approach using longitudinal health survey data in urban China. Soc. Sci. Med. 192, 74-84 (2017)

20. Oldrini, B., Curiel-García, Á., Marques, C., Matia, V., Uluçkan, Ö., Graña-Castro, O., Torres-Ruiz, R., Rodriguez-Perales, S., Huse, J.T., Squatrito, M.: Somatic genome editing with the RCAS-TVA-CRISPR-Cas9 system for precision tumor modeling. Nat. Commun. 9(1), 1-16 (2018)

21. Ashwood, M., Jerosch-Herold, C., Shepstone, L.: Learning to live with a hand nerve disorder: a constructed grounded theory. J. Hand Ther. 32(3), 334-344 (2019)

22. Hariharan, G.: Two reliable wavelet methods to Fitzhugh-Nagumo (FN) and fractional FN equations. In: Wavelet Solutions for Reaction-Diffusion Problems in Science and Engineering, pp. 135-146. Springer, Berlin (2019)

23. Gambino, G., Lombardo, M., Rubino, G., Sammartino, M.: Pattern selection in the 2D Fitzhugh-Nagumo model. Ric. Mat. 68(2), 535-549 (2019)

24. Namjoo, M., Zibaei, S.: Numerical solutions of Fitzhugh-Nagumo equation by exact finite-difference and NSFD schemes. Comput. Appl. Math. 37(2), 1395-1411 (2018)

25. Ori, H., Marder, E., Marom, S.: Cellular function given parametric variation in the Hodgkin and Huxley model of excitability. Proc. Natl. Acad. Sci. 115(35), E8211-E8218 (2018)

26. Khater, M.M., Attia, R.A., Baleanu, D.: Abundant new solutions of the transmission of nerve impulses of an excitable system. Eur. Phys. J. Plus 135(2), 1-12 (2020)

27. Khater, M.M., Attia, R.A., Abdel-Aty, A.-H., Abdel-Khalek, S., Al-Hadeethi, Y., Lu, D.: On the computational and numerical solutions of the transmission of nerve impulses of an excitable system (the neuron system). J. Intell. Fuzzy Syst. 38(3), 2603-2610 (2020)

28. Goufo, E.F.D., Kumar, S., Mugisha, S.: Similarities in a fifth-order evolution equation with and with no singular kernel. Chaos Solitons Fractals 130, 109467 (2020)

29. Ghanbari, B., Kumar, S., Kumar, R.: A study of behaviour for immune and tumor cells in immunogenetic tumour model with non-singular fractional derivative. Chaos Solitons Fractals 133, 109619 (2020)

30. Gao, F., Yang, X.-J., Ju, Y.: Exact traveling-wave solutions for one-dimensional modified Korteweg-de Vries equation defined on Cantor sets. Fractals 27(01), 1940010 (2019)

31. Rezazadeh, H., Korkmaz, A., Khater, M.M., Eslami, M., Lu, D., Attia, R.A.: New exact traveling wave solutions of biological population model via the extended rational sinh-cosh method and the modified Khater method. Mod. Phys. Lett. B 33(28), 1950338 (2019)

32. Attia, R.A., Lu, D., Ak, T., Khater, M.M.: Optical wave solutions of the higher-order nonlinear Schrödinger equation with the non-Kerr nonlinear term via modified Khater method. Mod. Phys. Lett. B 34, 2050044 (2020)

33. Khater, M.M., Lu, D., Attia, R.A.: Dispersive long wave of nonlinear fractional Wu-Whang system via a modified auxiliary equation method. AIP Adv. 9(2), 025003 (2019)

34. Osman, M., Lu, D., Khater, M.M.: A study of optical wave propagation in the nonautonomous Schrödinger-Hirota equation with power-law nonlinearity. Results Phys. 13, 102157 (2019)

35. Attia, R.A., Lu, D., Khater, M.M.A.: Chaos and relativistic energy-momentum of the nonlinear time fractional Duffing equation. Math. Comput. Appl. 24(1), 10 (2019)

36. Lu, D., Seadawy, A.R., Khater, M.M.: Structures of exact and solitary optical solutions for the higher-order nonlinear Schrödinger equation and its applications in mono-mode optical fibers. Mod. Phys. Lett. B 33(23), 1950279 (2019)

37. Khater, M.M., Lu, D., Attia, R.A.: Lump soliton wave solutions for the (2+1)-dimensional Konopelchenko-Dubrovsky equation and KdV equation. Mod. Phys. Lett. B 33(18), 1950199 (2019)

38. Kumar, S., Kumar, R., Cattani, C., Samet, B.: Chaotic behaviour of fractional predator-prey dynamical system. Chaos Solitons Fractals 135, $109811(2020)$

39. Kumar, S., Kumar, R., Agarwal, R.P., Samet, B.: A study of fractional Lotka-Volterra population model using Haar wavelet and Adams-Bashforth-Moulton methods. Math. Methods Appl. Sci. 43(8), 5564-5578 (2020)

40. Kumar, S., Ghosh, S., Samet, B., Goufo, E.F.D.: An analysis for heat equations arises in diffusion process using new Yang-Abdel-Aty-Cattani fractional operator. Math. Methods Appl. Sci. 43(9), 6062-6080 (2020)

41. Kumar, S., Ahmadian, A., Kumar, R., Kumar, D., Singh, J., Baleanu, D., Salimi, M.: An efficient numerical method for fractional SIR epidemic model of infectious disease by using Bernstein wavelets. Mathematics 8(4), 558 (2020)

42. Alshabanat, A., Jleli, M., Kumar, S., Samet, B.: Generalization of Caputo-Fabrizio fractional derivative and applications to electrical circuits. Front. Phys. 8, 64 (2020)

43. Baleanu, D., Jleli, M., Kumar, S., Samet, B.: A fractional derivative with two singular kernels and application to a heat conduction problem. Adv. Differ. Equ. 2020(1), 1 (2020)

44. Liu, J.-g., Yang, X.-j., Feng, Y.-y.: On integrability of the extended (3 + 1)-dimensional Jimbo-Miwa equation. Math. Methods Appl. Sci. 43(4), 1646-1659 (2020)

45. Liu, J.-G., Yang, X.-J., Feng, Y.-Y., Cui, P.: A new perspective to study the third order modified KdV equation on fracta set. Fractals (2020)

46. Liu, J.-G., Yang, X.-J., Feng, Y.-Y.: On integrability of the time fractional nonlinear heat conduction equation. J. Geom. Phys. 144, 190-198 (2019)

47. Yang, X.-J., Gao, F.: A new technology for solving diffusion and heat equations. Therm. Sci. 21 (1 Part A), 133-140 (2017)

48. Yang, X.-J.: A new integral transform operator for solving the heat-diffusion problem. Appl. Math. Lett. 64, 193-197 (2017)

49. Yang, X.-J., Feng, Y.-Y., Cattani, C., Inc, M.: Fundamental solutions of anomalous diffusion equations with the decay exponential kernel. Math. Methods Appl. Sci. 42(11), 4054-4060 (2019)

50. Chen, J., Ma, Z.: Consistent Riccati expansion solvability and soliton-cnoidal wave interaction solution of a ( $2+$ 1)-dimensional Korteweg-de Vries equation. Appl. Math. Lett. 64, 87-93 (2017)

51. Islam, N., Khan, K., Islam, M.H.: Travelling wave solution of Dodd-Bullough-Mikhailov equation: a comparative study between generalized Kudryashov and improved F-expansion methods. J. Phys. Commun. 3(5), 055004 (2019)

52. Bi, Q., Huang, J., Lu, Y., Zhu, L., Ding, H.: A general, fast and robust B-spline fitting scheme for micro-line tool path under chord error constraint. Sci. China, Technol. Sci. 62(2), 321-332 (2019)

53. Nazir, T., Abbas, M., Ismail, A.I.M., Majid, A.A., Rashid, A.: The numerical solution of advection-diffusion problems using new cubic trigonometric B-splines approach. Appl. Math. Model. 40(7-8), 4586-4611 (2016) 
54. Hasan, S., El-Ajou, A., Hadid, S., Al-Smadi, M., Momani, S.: Atangana-Baleanu fractional framework of reproducing kernel technique in solving fractional population dynamics system. Chaos Solitons Fractals 133, 109624 (2020)

55. Ghanbari, B., Günerhan, H., Srivastava, H.: An application of the Atangana-Baleanu fractional derivative in mathematical biology: a three-species predator-prey model. Chaos Solitons Fractals 138, 109910 (2020)

56. Khater, M.M., Attia, R.A., Abdel-Aty, A.-H., Abdou, M., Eleuch, H., Lu, D.: Analytical and semi-analytical ample solutions of the higher-order nonlinear Schrödinger equation with the non-Kerr nonlinear term. Results Phys. 16, 103000 (2020)

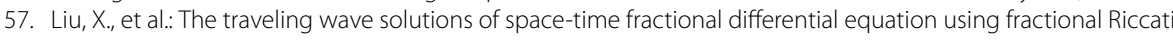
expansion method. J. Appl. Math. Phys. 6(10), 1957 (2018)

58. Kaur, B., Gupta, R.: Dispersion analysis and improved F-expansion method for space-time fractional differential equations. Nonlinear Dyn. 96(2), 837-852 (2019)

Submit your manuscript to a SpringerOpen ${ }^{\circ}$ journal and benefit from:

- Convenient online submission

- Rigorous peer review

- Open access: articles freely available online

- High visibility within the field

- Retaining the copyright to your article

Submit your next manuscript at $\boldsymbol{~ s p r i n g e r o p e n . c o m ~}$ 